\title{
Estudio paramétrico de la gasificación del coque de petróleo mexicano: efecto de la alimentación de coque de petróleo sobre las características energéticas del gas sintético (gassin)
}

\section{Parametric Study of Gasification of the Mexican Petroleum Coke: Effect Feed Petroleum Coke on Energetic Characteristic of the Synthetic Gas (syngas)}

\author{
González-Rocha J.C. \\ Instituto Tecnológico de Cd. Madero, Tamaulipas \\ E-mail:jcglezr_02@yahoo.com.mx \\ Urquiza-Beltrán G. \\ Centro de Investigación en Ingeniería y Ciencias Aplicadas \\ División de Turbomaquinaria \\ Universidad Autónoma del Estado de Morelos \\ E-mail:gurquiza@uaem.mx
}

\author{
Longoria-Ramírez R. \\ Departamento de Ingeniería Mecánica \\ Centro Nacional de Investigación y Desarrollo Tecnológico \\ (CENIDET) \\ E-mail:rlr@cenidet.edu.mx
}

Información del artículo: recibido: junio de 2009, aceptado: septiembre de 2010

\section{Resumen}

En este trabajo se realiza un estudio paramétrico de la gasificación del coque de petróleo mexicano de las refinerías de Cd. Madero, Tamaulipas denominado CRM y Cadereyta, Nuevo, León denominado CRC. Se evalúa el comportamiento energético del proceso de gasificación, con la finalidad de encontrar una alimentación de coque de petróleo de máximo provecho energético. Se analiza el efecto que tiene la alimentación de coque de petróleo sobre las características energéticas del gassin limpio (gasl): la composición molar de $\mathrm{CO} \mathrm{e} \mathrm{H}_{2}$, el poder calorífico superior $\left(\mathrm{PCS}_{\text {gasl }}\right.$ ), la potencia energética $\left(\Omega_{\text {gasl }}\right)$, la eficiencia de gas frío $\left(\mathrm{EGF}_{\text {gas }}\right)$ y la eficiencia térmica de gasificación $\left(\eta_{\mathrm{tg}}\right)$. El estudio paramétrico se realiza para una relación $0.64 \leq$ oxigeno/ coque $\leq 1.11$ manteniéndose constante el flujo de oxígeno $(6036.388 \mathrm{~kg} / \mathrm{h})$ y una relación vapor de agua/oxígeno de $23 \%$. Se observó que en la relación oxígeno/coque de 0.77 y 0.94 se presentan las mejores características energéticas del gasl del CRM y CRC, respectivamente. Las mejores características energéticas del gasl correspondientes al CRM y al CRC son: composición molar de CO $(65.6,68.5), \mathrm{H}_{2}(32.29,29.13), \mathrm{PCS}_{\text {gasl }}\left(14054.6 \mathrm{~kJ} / \mathrm{kg}_{\text {gasl }} 13438.76\right.$ $\left.\mathrm{kJ} / \mathrm{kg}_{\text {gas }}\right), \Omega_{\text {gasl }}(204.86 \mathrm{GJ} / \mathrm{h}, 178.02 \mathrm{GJ} / \mathrm{h}), \mathrm{EGF}_{\text {gasl }}(74.43 \%, 71.14 \%), \eta_{\mathrm{tg}}(86.2 \%$, $83.22 \%$ ). Al gasificar el coque de petróleo CRM la energía producida es a razón de $26.20 \mathrm{MJ} / \mathrm{kg}_{\text {coqué }}$ mientras que al gasificar el coque de petróleo CRC se producen a razón de $27.74 \mathrm{MJ} / \mathrm{kg}_{\text {coque }}$.

\section{Descriptores}

- composición molar

- poder calorífico

- potencia energética

- eficiencia de gas frío

- eficiencia térmica

- gasificación 


\begin{abstract}
In this work, a parametric study the gasification of the Mexican petroleum coke from the refineries of Cd Madero (CRM) and Cadereyta, Nuevo León (CRC) is performed. It is evaluates the energy behavior of the gasification process with objective to research which feedstock of petroleum coke has a better yield of energy. Effect feed petroleum coke on energy characteristics of the clear synthetic gas (gasl): molar composition of $\mathrm{CO}$ and $\mathrm{H}_{2}$, High Heating Value $\left(H H V_{\text {gasl }}\right)$, energy power $\left(\Omega_{\text {gass }}\right)$, Cold Gas Efficiency $\left(C G E_{\text {gasl }}\right)$ and thermal efficiency of gasification $\left(\eta_{t g}\right)$ is realized. The parametric study has been realized for $0.64 \leq$ oxygen/coke $\leq 1.11$ ratio with the flow of oxygen $(6036.388 \mathrm{~kg} / \mathrm{h})$ and steam/oxygen of $23 \%$ both constant. It is observed that in oxygen/coke 0.77 and 0.94 ratio it is present the best energy characteristics of the gasl from $C R M$ and $C R C$, respectively. The best energy characteristics relationship of the gasl fined are: molar composition $\mathrm{CO}(65.6,68.5), \mathrm{H}_{2}(32.29$, 29.13), HHV $V_{\text {gasl }}\left(14054.6 \mathrm{~kJ} / \mathrm{kg}_{\text {gasl }} 13438.76 \mathrm{~kJ} / \mathrm{kg}_{\text {gasl }}\right), \Omega_{\text {gasl }}(204.86 \mathrm{GJ} / \mathrm{h}, 178.02$ GJ/h), CGE $E_{\text {gasl }}(74.43 \%, 71.14 \%), \eta_{t g}(86.2 \%, 83.22 \%)$. The gasification of petroleum coke CRM produced energy with $26.20 \mathrm{MJ} / \mathrm{kg}_{\text {coque }}$ rates whereas that gasification of the petroleum coke CRC produced $27.74 \mathrm{MJ} / \mathrm{kg}_{\text {coque }}$ rates.
\end{abstract}

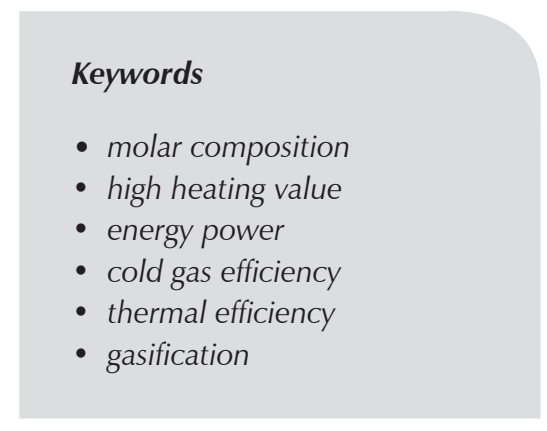

\section{Introducción}

A nivel mundial, en la última década continua incrementándose el interés por usar el coque del petróleo como combustible en la generación limpia de energía eléctrica aplicando la tecnología de gasificación (Holt, 1996), (Johnk et al., 1996), Generux et al., 1996), (Bryan et al, 1996), (Anthony et al., 1997), (Yañez, 2000), (Fernández et al., 2007), (González, 2007). Este interés se debe principalmente al incremento en la producción del coque de petróleo como consecuencia del procesamiento de mayores volúmenes de crudos pesados en las refinerías (González et al., 2001).

Actualmente en México, el Sistema Nacional de Refinación a través de sus refinerías en Cd. Madero, Tamaulipas y Cadereyta en Nuevo León produce en total 4500 toneladas por día (TPD) de coque de petróleo (González et al., 2008). Sin embargo, con la reconfiguración de las refinerías de Minatitlán, Ver., Salamanca, Gto. y la nueva refinería de Tula, Hidalgo (proyectada recientemente) se estima que para mediados de la siguiente década la producción de coque de petróleo se incremente en un 500\% (Pemex-refinación, 2009).

De acuerdo con las tendencias tecnológicas en los procesos de combustión (Fernández et al., 1998), (Secretaría de energía, 2007) y debido al alto contenido de azufre y metales como el vanadio y níquel, el coque de petróleo es considerado como un desecho de refinería, razón por la cual es barato y su tendencia en precio por unidad de energía permanecerá aproximadamente constante en las próximas dos décadas (figura 1).
De los estudios similares al presente sobre la gasificación del coque de petróleo se puede mencionar primeramente el realizado por Mahagaokar y Hauser en 1992. Ellos utilizaron una relación oxígeno/coque en el rango de 1.01 a 1.09 y generaron energía a razón de $29.13 \mathrm{MJ} / \mathrm{kg}_{\text {coque }}$ (Mega Joules por $\mathrm{kg}$ de coque) con una EGF de $78.6 \%$ en una relación oxígeno/coque de 1.02 utilizando un proceso de gasificación Shell (SCGP-1). Por su parte, Nagpal et al. en el 2004 simuló la gasificación del coque de petróleo, generando energía a razón de $25.61 \mathrm{MJ} / \mathrm{kg}_{\text {coque }}$ con una EGF de $78.9 \%$.

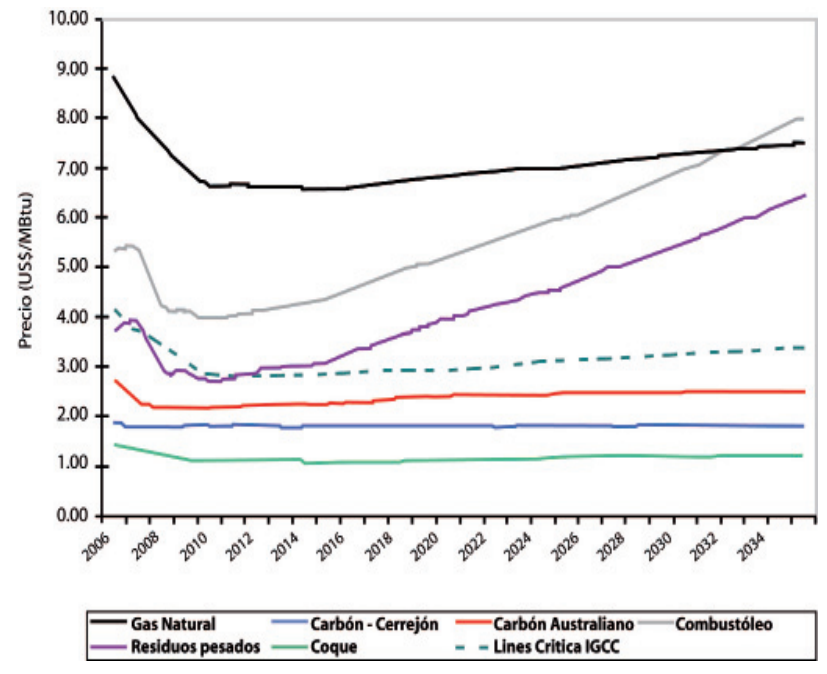

Figura 1. Estimación de la volatilidad de precios de los combustibles susceptibles a usarse (Fernández et al., 2008) 
Posteriormente, Hiu-Zou et al., en el 2006, realizó la gasificación del coque de petróleo con $\mathrm{CO}_{2}$. Ellos estudiaron la cinética de reacción de la gasificación del coque con base en su estructura porosa. Encontraron que la razón de gasificación es una función de distribución normal y la temperatura obedece a la ley de Arrhenius.

Un proceso de gasificación en ASPEN fue estructurado para gasificar el coque de petróleo mexicano de las refinerías de $\mathrm{Cd}$. Madero, Tamaulipas y Cadereyta, Nuevo León (González et al., 2008). El modelo fue utilizado para evaluar las características energéticas del gas sintético (gassin) para una relación oxígeno/coque de 1.02. Para iguales condiciones de operación en la gasificación del coque de petróleo de Cadereyta se genera energía a razón de $26.46 \mathrm{MJ} / \mathrm{kg}_{\text {coque }}$ mientras que al gasificar el coque de petróleo de Madero se generan 22.09 $\mathrm{MJ} / \mathrm{kg}_{\text {coque }}$ con una eficiencia de gas frío (EGF) del gasl de $67.87 \%$ y $62.75 \%$, respectivamente.

De lo anterior, se puede observar el particular interés por investigar la gasificación del coque de petróleo por diferentes medios y caracterizando el gas sintético (gassin) obtenido, el cual contiene valor energético para ser usado como combustible. Sin embargo, en los estudios anteriores no se ha realizado un estudio paramétrico del proceso de gasificación en función únicamente de la alimentación de coque de petróleo al reactor.

El objetivo del presente trabajo es encontrar, mediante la simulación del proceso de gasificación, las mejores características energéticas del gas sintético limpio (gasl): el poder calorífico superior $\left(\mathrm{PCS}_{\text {gas }}\right)$, la potencia energética $\left(\Omega_{\text {gas }}\right)$, la eficiencia de gas frío $\left(E_{\text {gas }}\right)$ y la eficiencia térmica de gasificación $\left(\eta_{\mathrm{tg}}\right)$; considerando como parámetro variacional la alimentación de coque de petróleo procedente de las plantas coquizadoras del país.

Asimismo, el conocimiento de estos parámetros resulta de importancia para estimar o evaluar la factibilidad y conveniencia de integrar un sistema de gasificación de los coques mexicanos a una planta de ciclo combinado para la generación limpia de electricidad, considerando a la tecnología de los ciclos combinados con gasificación integrada como una alternativa viable de implementarse en México.

Por otra parte, el coque de petróleo en conjunto con el oxígeno son reactivos de importancia económica, por lo tanto, se estima que una vez comercializado el gas sintético (gassin) producto de estos reactivos ambos incrementarán su precio y será necesario optimizar su consumo.

Para obtener los resultados de la gasificación del coque de petróleo mexicano se desarrolló un modelo de simulación en ASPEN PLUS SHELL, 2003; el cual se seleccionó por su gran versatilidad de modelos termodi- námicos y su gran flexibilidad para la simulación en interfaces con el usuario.

\section{Propiedades del coque de petróleo Mexicano}

El coque de petróleo mexicano es un producto residual sólido de alto contenido energético, alto índice de pulverización, bajo en volátiles, alto contenido de azufre (alrededor de un $7 \%$ ) y metales como el vanadio y níquel. Contiene un poder calorífico entre $30000 \mathrm{~kJ} / \mathrm{kg}$ y $40000 \mathrm{~kJ} / \mathrm{kg}$ dependiendo de las exigencias de refinación de los productos de valor agregado como la gasolina, turbosina, diesel, etc. (Millán et al., 1992), (Mahagaokar et al., 1992), (Cook et al., 1995). Sin embargo, a pesar del alto contenido energético del coque de petróleo comparado con un carbón subituminoso mexicano, éste no puede usarse como combustible en plantas de potencia convencionales (carboeléctricas o termoeléctricas), debido a su alto contenido de azufre y metales como el vanadio y el níquel, a menos que estas plantas de potencia sean equipadas con potentes depuradores de gases contaminantes.

El análisis próximo (tal como se recibe), el análisis último (libre de humedad) y el poder calorífico superior del coque de petróleo $\left(\mathrm{PCS}_{\text {coque }}\right)$ producido en las refinerías de $\mathrm{Cd}$. Madero y Cadereyta y las normas ASTM (American Society for Testing Materials, por sus siglas en inglés) que se aplicaron para obtener sus valores se presentan en la tabla 1.

\section{Gasificación}

La gasificación es una tecnología que en la última década ha resultado de gran aceptación para convertir com-

Tabla 1. Composición del coque CRM y CRC, (\% peso) (González et al., 2008)

\begin{tabular}{cccc}
\hline Análisis próximo & CRM & CRC & Norma ASTM \\
\hline Humedad & 10.54 & 1.21 & D3173-03 \\
Cenizas & 0.35 & 0.36 & D3174-02 \\
Materia volátil & 10.22 & 10.65 & D3175-02 \\
Carbón fijo & 78.89 & 87.78 & D3172-R02 \\
& & & \\
Análisis último & & & \\
Cenizas & 0.35 & 0.36 & D3174-02 \\
Carbono & 84.24 & 84.63 & D5373-02 \\
Hidrógeno & 3.90 & 3.91 & D5373-02 \\
Nitrógeno & 0.79 & 0.72 & D5373-02 \\
Azufre & 6.04 & 5.91 & D129-00 \\
Oxígeno & 4.68 & 4.47 & \\
PCS $_{\text {coque }}(\mathrm{kJ} / \mathrm{kg})$ & 35202.61 & 38987.48 & D5865-03 \\
\hline
\end{tabular}


bustibles sólidos como el carbón, biomasa y últimamente el coque de petróleo en un gas sintético. Básicamente consiste en convertir un combustible sólido considerado como contaminante en un gas limpio, libre hasta en un $99 \%$ de compuestos de azufre $\left(\mathrm{H}_{2} \mathrm{~S}, \mathrm{COS}\right)$ y metales pesados como vanadio y níquel.

La reacción básica de gasificación puede expresarse como sigue:

$\mathrm{C}_{\mathrm{n}} \mathrm{H}_{\mathrm{m}}+\mathrm{n} / 2 \mathrm{O}_{2} \Rightarrow \mathrm{nCO}+\mathrm{m} / 2 \mathrm{H}_{2}$

La composición del gas resultante (gassin) está formado principalmente de $\mathrm{CO}_{\text {y }} \mathrm{H}_{2}$ (alrededor de $90 \%$ ) y el resto lo conforman $\mathrm{CO}_{2}, \mathrm{H}_{2} \mathrm{O}, \mathrm{CH}_{4}, \mathrm{~N}_{2}, \mathrm{H}_{2} \mathrm{~S}$ y COS.

Desde hace dos décadas, países como Estados Unidos de América, Holanda, España, Italia, Japón, Taiwan, entre otros, usan el coque de petróleo, la tecnología de gasificación y los ciclos combinados para generar electricidad y vapor aplicando la nueva generación de plantas de potencia denominadas ciclos, combinado con gasificación integrada (IGCC, por sus siglas en inglés); (Northridge, 1986); (Fabela, 1998); (Fernández et al., 1995); (Bolado, 1995); (Ahman et al., 2002); (EPRI, 2006); (Fernández et al., 2007); (González, 2007).

\section{Modelo termodinámico de gasificación}

Se estructuró un modelo de gasificación, el cual fue validado para su aplicación con el modelo de gasificación Shell SCGP-1 utilizado por Mahagaokar y Hauser (1992) para gasificar el coque de petróleo americano. En ambos modelos se utilizo un gasificador de flujo por arrastre.

En la simulación del proceso de gasificación de coque de petróleo mexicano se consideró un gasificador de flujo por arrastre, operando a una temperatura de $1515{ }^{\circ} \mathrm{C}$ y presión de 40 bar. De acuerdo con los resultados de la simulación, el efecto de la presión resultó ser de muy limitada influencia sobre las características energéticas del gassin, en tanto que para la temperatura se tomó en cuenta que la velocidad de reacción para las reacciones de combustión que se dan más adelante, son limitadas por la transferencia de masa a temperaturas mayores de $1150^{\circ} \mathrm{C}$, no así la cinética de gasificación de vapor de agua y dióxido de carbono a temperaturas mayores a $1400^{\circ} \mathrm{C}$ (Hui et al., 2006).

Durante un proceso de gasificación se desarrollan una serie de reacciones químicas que dependen de las condiciones de operación del gasificador. Las principales reacciones químicas involucran al carbono, monóxido de carbono, dióxido de carbono, hidrógeno, vapor de agua, metano, ácido sulfhídrico y sulfuro de carbo- nilo. Estas reacciones pueden expresarse de la siguiente manera (Himan et al., 2003):

$$
\begin{array}{ll}
2 \mathrm{C}+\mathrm{O}_{2} \Rightarrow 2 \mathrm{CO} & -111 \mathrm{~kJ} / \mathrm{mol} \\
2 \mathrm{CO}+\mathrm{O}_{2} \Rightarrow 2 \mathrm{CO}_{2} & -393 \mathrm{~kJ} / \mathrm{mol} \\
2 \mathrm{H}_{2}+\mathrm{O}_{2} \Rightarrow 2 \mathrm{H}_{2} \mathrm{O} & -242 \mathrm{~kJ} / \mathrm{mol} \\
\mathrm{C}+\mathrm{CO}_{2} \Leftrightarrow 2 \mathrm{CO} & +172 \mathrm{~kJ} / \mathrm{mol} \\
\mathrm{C}+\mathrm{H}_{2} \mathrm{O} \Leftrightarrow \mathrm{CO}+\mathrm{H}_{2} & +131 \mathrm{~kJ} / \mathrm{mol} \\
\mathrm{C}+2 \mathrm{H}_{2} \Leftrightarrow \mathrm{CH}_{4} & -75 \mathrm{~kJ} / \mathrm{mol}
\end{array}
$$

Esencialmente las ecuaciones 2, 5, 6 y 7 representan reacciones heterogéneas que determinan la composición del gassin en el estado de equilibrio. Estas cuatro reacciones describen la manera en que el coque de petróleo puede ser gasificado. La reacción 5 produce el $\mathrm{CO}$ al gasificar el carbono en una atmósfera de $\mathrm{CO}_{2}$, la reacción 6 es predominante sobre el proceso gas-agua y la reacción 7 es la base de hidrogenación en el proceso de gasificación. Obsérvese que en las ecuaciones 5-7 se encuentran involucradas las reacciones homogéneas del CO y la reacción de metanación con vapor de agua, ecuaciones 8 y 9 .

$$
\begin{array}{ll}
\mathrm{CO}+\mathrm{H}_{2} \mathrm{O} \Leftrightarrow \mathrm{CO}_{2}+\mathrm{H}_{2} & -41 \mathrm{~kJ} / \mathrm{mol} \\
\mathrm{CH}_{4}+\mathrm{H}_{2} \mathrm{O} \Leftrightarrow \mathrm{CO}_{2}+3 \mathrm{H}_{2} & +206 \mathrm{~kJ} / \mathrm{mol}
\end{array}
$$

En particular, la reacción de equilibrio del $\mathrm{CO}$ con vapor de agua, ecuación 8, es una reacción importante, si se considera que interviene en el control de la composición del gassin limpio (gasl); a temperaturas mayores de $1000{ }^{\circ} \mathrm{C}$ su velocidad de reacción es tan rápida que puede considerarse que se encuentra en equilibrio (Hui et al., 2006), (Nagpal et al., 2004) como es el caso de este trabajo de simulación.

Las ecuaciones 5 a 9 son reacciones reversibles que se realizan simultáneamente a velocidades diferentes.

En general, toda mezcla reactiva a temperatura y presión determinada se desarrolla en la dirección del decrecimiento de la función de Gibbs (G) bajo el siguiente criterio de equilibrio químico (Cengel et al., 1998); (Bejan, 1980).

$(d G)_{T, P}=0$ 
Es decir, cuando la función de Gibbs es mínima, la reacción química se para y se establece el equilibrio químico. Es necesario especificar que una reacción química a una temperatura y presión constantes no puede desarrollarse en dirección del incremento de la función de Gibbs, debido a que se violaría la segunda ley de la termodinámica

$d S \geq \frac{\delta Q}{T}$

Al resolver para cada reacción desde la 5 a 7, involucrando la 8 y 9 se puede obtener el criterio de equilibrio químico siguiente:

$\sum_{p} v_{i} \bar{g}-\sum_{r} v_{i} \bar{g}=0$

donde

$v$ es el coeficiente estequiométrico

$\bar{g}$ es la función de Gibbs molar

$\mathrm{p}$ representa a los productos de gasificación

$r$ representa a los reactivos involucrados

i iesimo producto o reactivo en la reacción química.

Las constantes de equilibrio $(K)$ de las reacciones involucradas en la gasificación se obtuvieron usando la correlación

$$
\operatorname{Ln}\left(K_{p, T}\right)=\operatorname{Ln}\left(K_{p, T_{0}}\right)+f(T)
$$

Considerando la ecuación 13 es posible encontrar las fracciones molares en el estado de equilibrio del gasl, obtenido mediante la gasificación del coque de petróleo mexicano (Himan et al., 2003).

\section{Resultados y discusión}

En este análisis paramétrico se evalúa el efecto de la alimentación de coque de petróleo expresados por la relación oxígeno/coque con el objetivo de buscar ¿Cuál relación oxígeno/coque?, para cada coque en particular se obtiene un gasl con las máximas características energéticas ( $\mathrm{PCS}_{\text {gasl }} \Omega_{\text {gasl }} \mathrm{EGF}_{\text {gasl }}, \eta_{\text {tg }}$ y composición quími$\mathrm{ca})$, esto, con la finalidad de obtener el mayor provecho de cada coque (CRM ó CRC) gasificado por separado. El análisis paramétrico se realiza para una alimentación de vapor de agua constante de $1385.369 \mathrm{~kg} / \mathrm{h}$ y para un rango de alimentación de coque de petróleo de 5418.304 a $9418.304 \mathrm{~kg} / \mathrm{h}$, una alimentación de oxígeno de $6036.388 \mathrm{~kg} / \mathrm{h}$, una presión de 42 bar y una temperatura de gasificación de $1515^{\circ} \mathrm{C}$.
Con la finalidad de tener un punto inicial y poder observar el comportamiento energético del gassin limpio (gasl): en este estudio paramétrico se considera de referencia la relación másica oxígeno/coque de 1.02, la cual fue usada por Mahagaokar y Hauser (1992) y considerada por González et al. (2008); para la validación y realización de un estudio preliminar de la gasificación de coque de petróleo mexicano. La comparación de las características energéticas de ambos coques gasificados por separado se realiza en condiciones iguales de operación.

\section{Composición química del gasI}

En la figura 1 se puede observar que, si la alimentación de coque en ambos se reduce a $5418.304 \mathrm{~kg} / \mathrm{h}$ correspondiente a una relación $\mathrm{O}_{2} /$ coque de 1.114, el porcentaje molar de CO presente en el gasl del CRC es de 67.23, mientras que para el CRM es de 62.93. Esta diferencia se debe principalmente a la influencia del carbono fijo $(\mathrm{CF})$ y humedad $(\mathrm{H})$ contenido en el correspondiente coque de petróleo gasificado (tabla 1).

Sin embargo, cuando la alimentación de coque se incrementa a $6418.304 \mathrm{~kg} / \mathrm{h}$ para ambos coques, el porcentaje molar de CO para el CRC tiene su máximo con un contenido de $68.52 \%$, mientras que el porcentaje molar de CO para CRM es de $64.78 \%$ correspondiente a una relación oxígeno/coque para ambos de 0.94 .

En la relación 0.94 el contenido de $\mathrm{H}_{2}$ para el CRC es de $29.13 \%$ molar y para el CRM de $28.73 \%$ molar (figura 2). La composición de $\mathrm{CO}_{\text {e } \mathrm{H}_{2}}$ para el CRC se considera una composición máxima alcanzada en la relación oxigeno/coque de 0.940 .

\section{Potencia energética del gasl}

En la relación oxígeno/coque de 0.94 el poder calorífico del gasl es de $13438.756 \mathrm{~kJ} / \mathrm{kg}_{\text {gasl }}$ para el CRC y de $12406.975 \mathrm{~kJ} / \mathrm{kg}$ de gasl del CRM con una diferencia de $1031.78 \mathrm{~kJ}$ por cada $\mathrm{kg}$ de gasl producido. La potencia energética del gasl se tiene $178.019 \mathrm{GJ} / \mathrm{h}$ para el CRC y $150.453 \mathrm{GJ} / \mathrm{h}$ para el CRM, es decir, la potencia energética del CRC es mayor en $27.566 \mathrm{GJ} / \mathrm{h}$, con respecto a la potencia energética del CRM. Por consiguiente, para iguales condiciones de alimentación de coque de $6418.304 \mathrm{~kg} / \mathrm{h}$ al gasificar el CRC se obtiene un incremento de energía de $4295 \mathrm{~kJ}$ por cada $\mathrm{kg}$ de coque gasificado (figuras 3 y 4). En esta relación oxígeno/coque de 0.94 el CRC produce $2.23826 \mathrm{~kg}_{\text {gas }} / \mathrm{kg}_{\text {coque }}$ mientras que para la gasificación del CRM se producen 2.04897 $\mathrm{kg}_{\text {gas }} / \mathrm{kg}_{\text {coque }}$. 
Eficiencia de gas frío del gasl $\left(\mathrm{EGF}_{\text {gasl }}\right)$

La eficiencia del gas frío del gasl es un parámetro de rendimiento, el cual se define como la cantidad de energía contenida en el gas limpio respecto a la energía del coque alimentado, es decir, es el porcentaje de energía del coque alimentado todavía presente en el gassin limpio. De acuerdo con esta definición, la EGF del gasl será sensible a las variaciones de la relación oxígeno/coque. Así, para una relación de oxígeno/coque de 0.94 se tiene el mayor contenido de energía del coque aún presente en el gassin limpio (gasl) con una EGF de $71.14 \%$ para la gasificación del CRC, mientras que EGF para la gasificación del CRM es de $66.59 \%$ con una diferencia de 4.55 puntos porcentuales, por consiguiente, la relación 0.940 puede ser considerada máxima para la gasificación del CRC (figura 5).

Si la alimentación del coque continua incrementándose, por ejemplo, en el intervalo en donde la relación

$0.77 \leq \frac{\mathrm{O}_{2}}{\text { coque }} \leq 0.940$ correspondiente a una alimentación

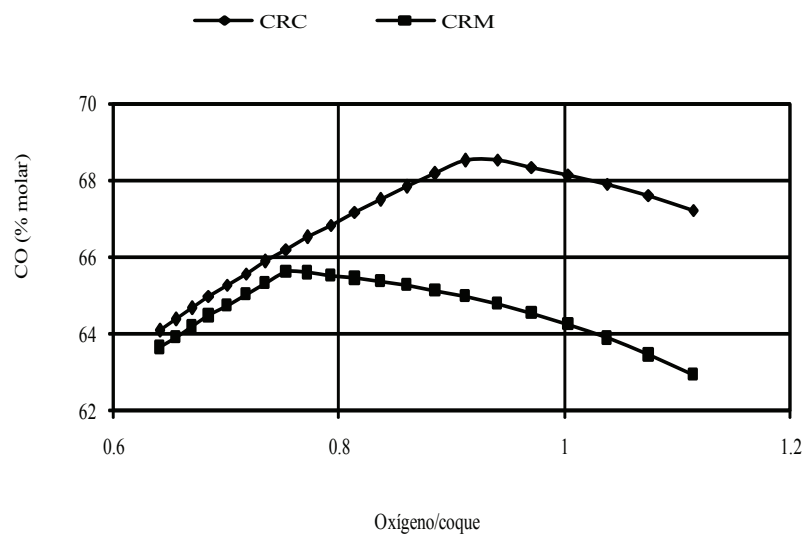

Figura 1. Variación del \% molar del $\mathrm{CO}$ en el gasl con respecto a la relación $\mathrm{O}_{2} /$ coque

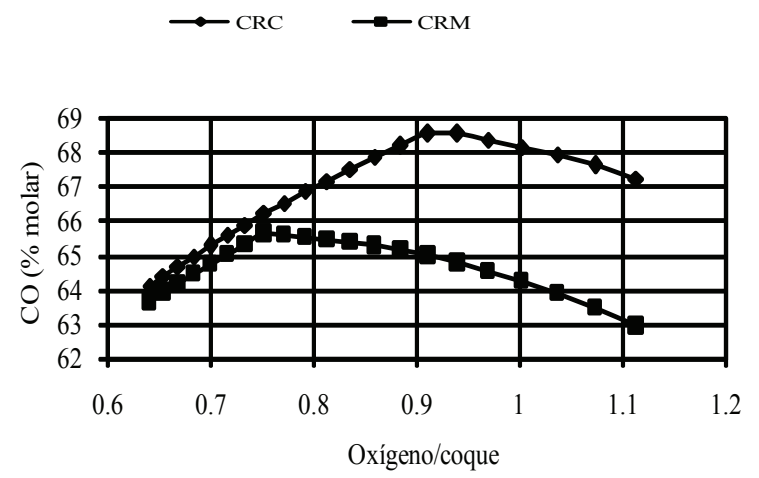

Figura 3. Variación del PCS del gasl con respecto a la relación $\mathrm{O}_{2}$ /coque de coque de petróleo de $6418.3042 \mathrm{~kg} / \mathrm{h}$ a $7818.304 \mathrm{~kg} / \mathrm{h}$, es decir, un incremento de $1400 \mathrm{~kg}$ de coque de petróleo al gasificador. El porcentaje molar de $\mathrm{CO}$ en el gasl del CRM aumenta de 64.78 a $65.60 \%$ molar, mientras que el porcentaje molar de CO del CRC disminuye de 68.52 a 66.51. El $\mathrm{H}_{2}$ toma relativa importancia en el valor del poder calorífico y como consecuencia en la potencia energética y $\mathrm{EGF}_{\text {gasl }}$.

Para este intervalo de alimentación de coque, el gasl del CRM presenta una gran mejora en sus características energéticas, alcanzando su máxima $\mathrm{EGF}_{\text {gasl }}$ de $74.43 \%$ en la relación oxígeno/coque de 0.77 , comparada con la $\mathrm{EGF}_{\text {gasl }}$ del CRC con un valor de $61.98 \%$ para igual alimentación de coque de $7818.304 \mathrm{~kg} / \mathrm{h}$. En esta relación la potencia energética del gasl del CRM es de $204.859 \mathrm{GJ} / \mathrm{h}$, un poder calorífico de $14054.565 \mathrm{~kJ} / \mathrm{kg}_{\text {gasl }}$, un porcentaje molar de $\mathrm{CO}$ de 65.6 y un porcentaje molar de $\mathrm{H}_{2}$ de 32.288; más allá de esta relación (menores de 0.77 ) el poder calorífico y la potencia energética se incrementa a expensas de un incremento en la alimentación del coque de petróleo, lo que sería antieconómico de acuerdo a la tendencia de la $\mathrm{EGF}_{\text {gasl }}$ en conjunto con el PCS del coque alimentado (figuras 1 a 6).



Figura 2. Variación del \% molar del $\mathrm{H}_{2}$ en el gasl con respecto a la relación $\mathrm{O}_{2} /$ coque

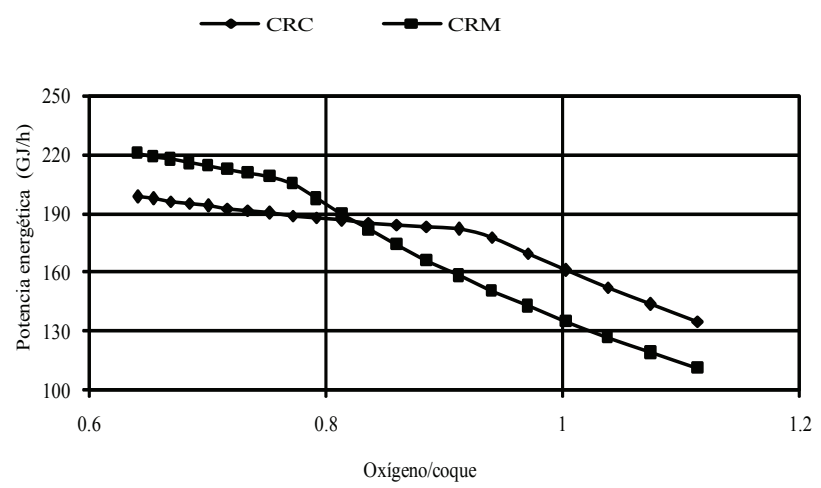

Figura 4. Variación de la potencia energética del gasl con respecto a la relación $\mathrm{O}_{2} /$ coque 


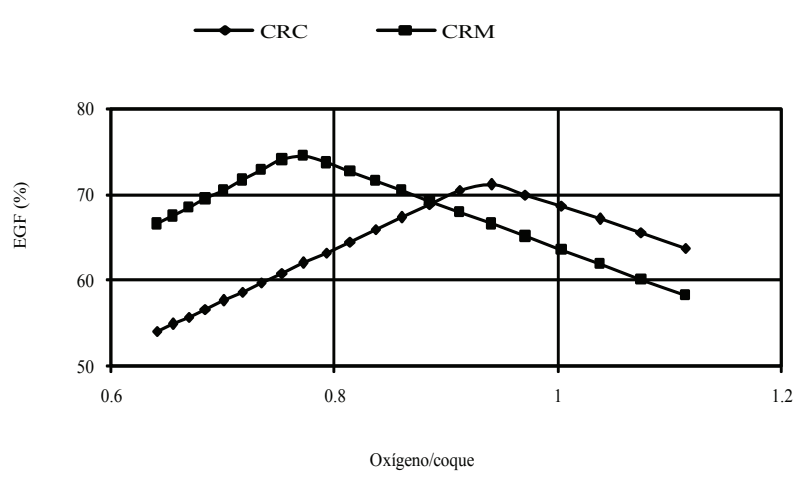

Figura 5. Variación de la EGF del gasl con respecto a la relación $\mathrm{O}_{2} /$ coque

En la misma relación oxígeno/coque igual a 0.77 las condiciones energéticas y la composición química del gasl del CRC adquieren valores de $66.51 \%$ de $\mathrm{CO}$, $31.240 \%$ molar de $\mathrm{H}_{2}$, PCS de $13880.165 \mathrm{~kJ} / \mathrm{kg}_{\text {gasl }}$ una $\Omega_{\text {gasl }}$ de $188.940 \mathrm{GJ} / \mathrm{h}$ y una $\mathrm{EGF}_{\text {gasl }}$ de $61.98 \%$.

Es decir, las características energéticas del CRM son importantes para altas alimentaciones de coque en el rango de $0.641 \leq \mathrm{O}_{2} /$ coque $\leq 0.77$ correspondiente a una alimentación de CRM entre $7818.304 \mathrm{~kg} / \mathrm{h}$ y 9418.304 $\mathrm{kg} / \mathrm{h}$, en donde el poder calorífico superior $\left(\mathrm{PCS}_{\text {gasl }}\right)$, potencia energética del gasl $\left(\Omega_{\text {gasl }}\right)$ y la $\mathrm{EGF}_{\text {gasl }}$ son mayores que las correspondientes al CRC, (figuras 3 a 5). Cabe resaltar que para este incremento de $2000 \mathrm{~kg} / \mathrm{h}$ de coque CRM y CRC $(7818.304 \mathrm{~kg} / \mathrm{h}$ a $9418.304 \mathrm{~kg} / \mathrm{h})$, el comportamiento de la $\mathrm{EGF}_{\text {gas' }}$ es tal que tiende a disminuir aproximadamente proporcional para ambas gasificaciones de coque de $74.43 \%$ a $66.55 \%$ para el CRM y de $61.98 \%$ a $54.04 \%$ para el CRC, (figura 5). Cabe hacer mención que los valores de EGF $_{\text {gasl }}$ y composición son coherentes con los valores reportados por encontradas por (Mahagaokar et al., 1992); (Nagpal et al., 2004); (Hiu et al., 2006) y (González, 2007 y 2008).

En estos resultados se observa que en la relación oxígeno/coque de 0.77 , cuando se gasifica el CRM se producen $2.462863 \mathrm{~kg}_{\text {gas }} / \mathrm{kg}_{\text {coque }}$ mientras que para la misma relación cuando se gasifica el CRC se producen $2.3 \mathrm{~kg}_{\text {gas }} / \mathrm{kg}_{\text {coque }}$ esta generación de gasl comparado con el gasl producido por el CRC para la relación 0.94 son mayores, principalmente para el CRM en $0.22603 \mathrm{~kg}_{\text {gas }} /$ $\mathrm{kg}_{\text {coque }}$.

\section{Eficiencia térmica de gasificación}

La eficiencia térmica de gasificación $\left(\eta_{\mathrm{tg}}\right)$ se define como la relación entre la suma de la potencia energética del gassin crudo (gassin inmediatamente a la salida del gasificador) obtenido y la potencia energética del vapor producido, respecto a la potencia energética del coque

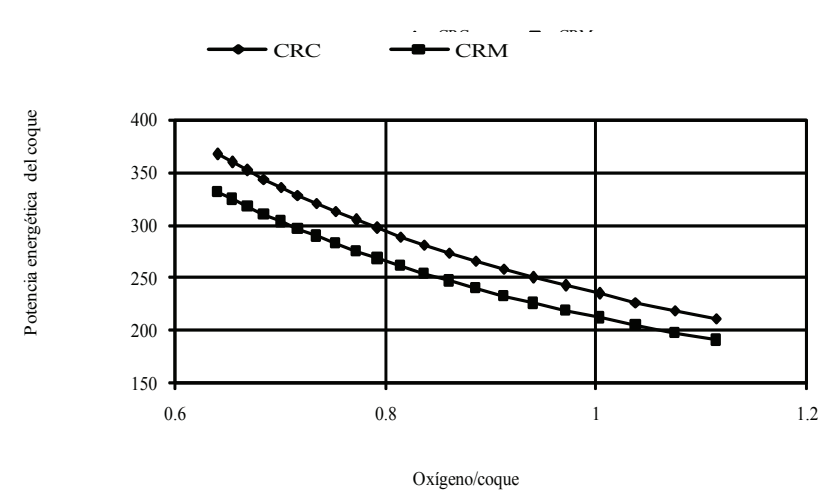

Figura 6. Potencia energética del coque de petróleo alimentado $(\mathrm{GJ} / \mathrm{h})$

alimentado. El sistema de gasificación produce vapor saturado disponible para algún uso futuro. La alimentación de coque al sistema de gasificación representada por la relación oxígeno/coque, la producción de gassin y la generación de vapor se relacionan de tal menara que tienen influencia directa sobre la eficiencia térmica de gasificación.

Cuando la relación oxígeno/coque se reduce de 0.940 a 0.77 correspondiente a un incremento en la alimentación de coque de $6418.304 \mathrm{~kg} / \mathrm{h}$ a $7818.304 \mathrm{~kg} / \mathrm{h}$ la potencia energética del gasc $\left(\Omega_{\text {gasc }}\right)$ del CRC se incrementa $15 \mathrm{GJ} / \mathrm{h}$ de $192.78 \mathrm{GJ} / \mathrm{h}$ a 207.6, mientras que la potencia energética del gasc $\left(\Omega_{\text {gasc }}\right)$ del CRM se incrementa $55.66 \mathrm{GJ} / \mathrm{h}$ de 164.91 a $220.57 \mathrm{GJ} / \mathrm{h}$, asimismo, la potencia energética del vapor saturado $\left(\Omega_{\text {vapor }}\right)$ del CRC varia de $15.52 \mathrm{GJ} / \mathrm{h}$ a $15.94 \mathrm{GJ} / \mathrm{h}$ y la del CRM de 15.60 $\mathrm{GJ} / \mathrm{h}$ a $16.63 \mathrm{GJ} / \mathrm{h}$ durante el intervalo considerado (figuras 8 y 9).

Por consiguiente, la eficiencia térmica del CRM se incrementa de $79.89 \%$ a $86.18 \%$ para una potencia energética del coque alimentado de $225.94 \mathrm{GJ} / \mathrm{h}$ a 275.22 , mientras que la eficiencia térmica del CRC disminuye de $83.22 \%$ a $73.34 \%$ para una potencia energética del coque alimentado de $250.23 \mathrm{GJ} / \mathrm{h}$ a $304.82 \mathrm{GJ} / \mathrm{h}$ de acuerdo con las figuras $6,7,8,9$ y tabla 2. Las relaciones oxígeno/coque de 0.94 y 0.77 son las relaciones donde el CRC y el CRM, respectivamente, alcanzan su máxima eficiencia térmica con un valor de $83.22 \%$ y $86.18 \%$, la primera para una alimentación de coque CRC de $6418.304 \mathrm{~kg} / \mathrm{h}$ y la segunda para una alimentación de coque de CRM de $7818.304 \mathrm{~kg} / \mathrm{h}$.

Cuando la relación oxígeno/coque se incrementa de 0.940 a 1.114 significa que la alimentación de coque se reduce de $6418.304 \mathrm{~kg} / \mathrm{h}$ a $5418.304 \mathrm{~kg} / \mathrm{h}$, dando como resultado una baja en la energía contenida en el gassin crudo, esto propicia que menos vapor sea generado y los flujos másicos del gassin crudo y vapor sean reducidos, disminuyendo así, la potencia energética del gas- 
sin crudo y la potencia energética del vapor y por consiguiente la eficiencia térmica de gasificación. Cuando se gasifica el CRC para una relación oxígeno/coque de 0.940 se alcanza una $\eta_{\mathrm{tg}}$ igual a $83.22 \%$, mientras que cuando se gasifica el CRM para igual relación oxígeno/ coque se obtiene una $\eta_{\mathrm{tg}}$ igual a $79.89 \%$ con una diferencia de 3.33 puntos porcentuales, (figura 7).

Un condensado comparativo de estos resultados puede ayudar a visualizar mejor las características energéticas del gasl analizadas anteriormente (tabla 2).

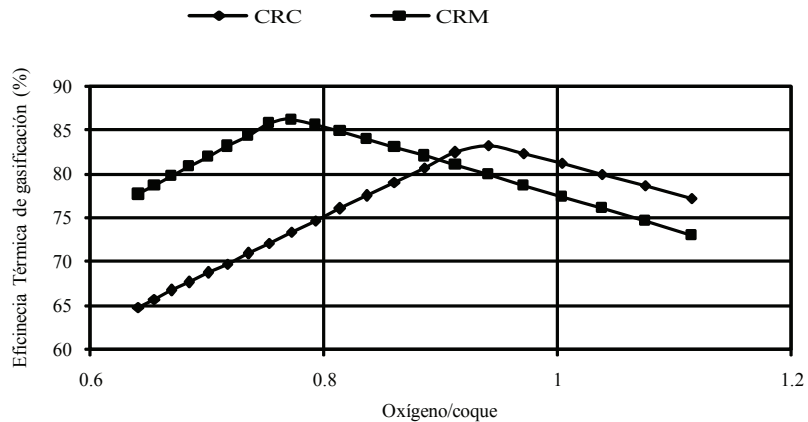

Figura 7. Variación de la eficiencia térmica con respecto a la relación $\mathrm{O}_{2}$ /coque

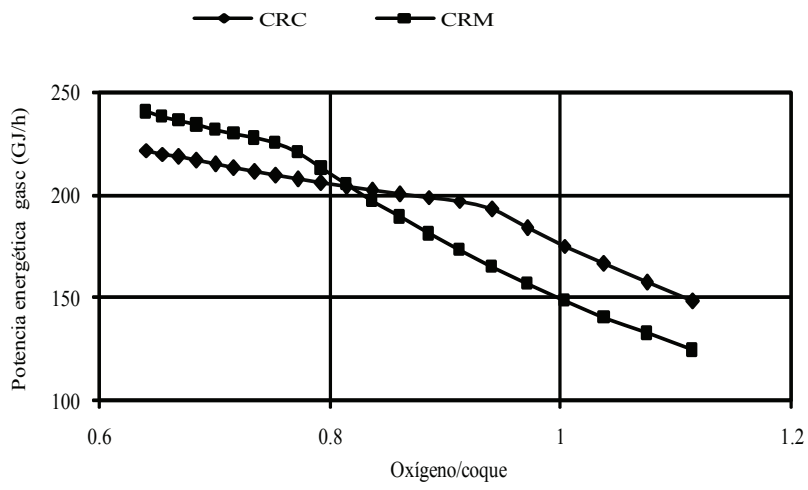

Figura 8. Variación de la $\Omega_{\text {gasc }}$ del CRC y del CRM con respecto a la relación $\mathrm{O}_{2} /$ coque

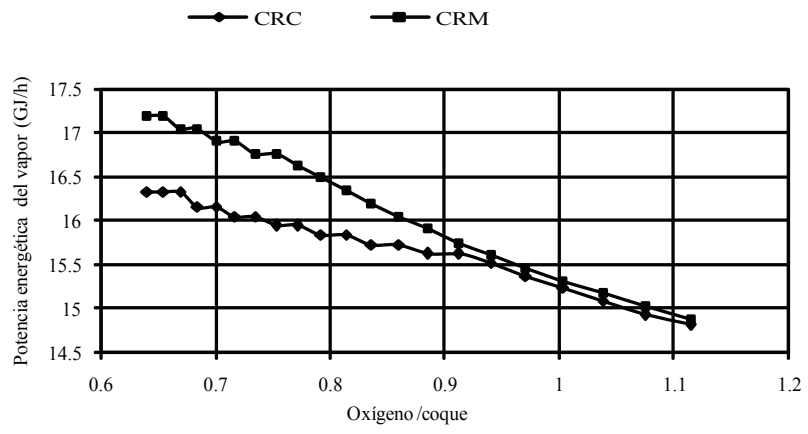

Figura 9. Variación de la potencia energética del vapor de gasificación con respecto a la relación $\mathrm{O}_{2} /$ coque
Tabla 2. Resumen de las características energéticas en la gasificación del CRC y CRM

\begin{tabular}{ccccc}
\hline & \multicolumn{2}{c}{$\mathrm{O}_{2} /$ coque $^{2}$} & 0.94 & \multicolumn{2}{c}{$\mathrm{O}_{2} /$ coque } & $=0.77$ \\
\hline & CRC max. & $\mathrm{CRM}$ & $\mathrm{CRC}$ & $\mathrm{CRM} \mathrm{max}$ \\
\hline $\mathrm{m}_{\text {coque }}(\mathrm{kg} / \mathrm{h})$ & 6418.304 & 6418.304 & 7818.304 & 7818.304 \\
$\mathrm{~m}_{\text {O2 }}(\mathrm{kg} / \mathrm{h})$ & 6036.388 & 6036.388 & 6036.388 & 6036.388 \\
$\mathrm{CO}(\% \mathrm{~mol})$ & 68.520 & 64.780 & 66.514 & 65.586 \\
$\mathrm{H}_{2}(\% \mathrm{~mol})$ & 29.132 & 28.726 & 31.240 & 32.288 \\
$\mathrm{PCS}_{\text {gasl }}(\mathrm{kJ} / \mathrm{kg})$ & 13438.756 & 12406.975 & 13880.165 & 14054.565 \\
$\Omega_{\text {gasl }}(\mathrm{GJ} / \mathrm{h})$ & 178.019 & 150.453 & 188.940 & 204.859 \\
$\Omega_{\text {coque }}(\mathrm{GJ} / \mathrm{h})$ & 250.23 & 225.94 & 304.82 & 275.22 \\
$\mathrm{EGF}_{\text {gasl }}(\%)$ & 71.14 & 66.59 & 61.98 & 74.43 \\
$\eta_{\text {th }}(\%)$ & 83.22 & 79.89 & 73.33 & 86.18 \\
\hline
\end{tabular}

\section{Conclusiones}

Para la próxima década, México pretende incrementar la producción de coque de petróleo en un $500 \%$ mediante la reconfiguración de las refinerías de Minatitlán, Salamanca, Tula, además de las refinerías de Madero y Cadereyta, las cuales pertenecen al sistema nacional de refinación.

Se encontraron dos relaciones de oxígeno/coque 0.77 y 0.94 correspondientes a una alimentación de coque de petróleo CRM de $7818.304 \mathrm{~kg} / \mathrm{h}$ y CRC de $6418.304 \mathrm{~kg} / \mathrm{h}$, respectivamente, en donde el gassin limpio (gasl) producido presenta las máximas características energéticas.

El rendimiento del coque de petróleo CRM es de $2.46 \mathrm{~kg}_{\text {gas }} / \mathrm{kg}_{\text {coque }}$ mientras que el rendimiento del CRC es de $2.24 \mathrm{~kg}_{\text {gas }} / \mathrm{kg}_{\text {coque }}$ correspondientes a una producción de $4.05 \mathrm{~kg}_{\text {gasl }} / \mathrm{seg}$ y $3.68 \mathrm{~kg}_{\text {gas }} / \mathrm{seg}$, respectivamente.

La producción de energía por kg de coque alimentado es: para el CRM $26.14 \mathrm{MJ} / \mathrm{kg}_{\text {coque }}$ y para el CRC 27.68 $\mathrm{MJ} / \mathrm{kg}_{\text {coque }}$.

Para una relación de oxígeno/coque de 0.94 se tiene una EGF de $66.95 \%$ para la gasificación del CRM, mientras que en la misma relación de 0.94 la EGF para la gasificación del CRC es de $71.14 \%$ con una diferencia de 4.55 puntos porcentuales, por consiguiente, la relación 0.940 puede ser considerada máxima para la gasificación del CRC.

El gasl del CRM presenta una gran mejora en sus características energéticas, alcanzando su máxima $\mathrm{EGF}_{\text {gasl }}$ de $74.43 \%$ en la relación oxígeno/coque de 0.77 comparada con la $\mathrm{EGF}_{\text {gasl }}$ del CRC con un valor de $61.98 \%$ para igual alimentación de coque de 7818.304 $\mathrm{kg} / \mathrm{h}$. 
Para valores de la relación oxígeno/coque menores a 0.77 para el CRM y menores a 0.94 para el CRC: el poder calorífico y la potencia energética se incrementa a expensas de un incremento en la alimentación del coque de petróleo, lo que sería antieconómico de acuerdo a la tendencia de la EGF $_{\text {gasl }}$ en conjunto con el PCS del coque alimentado.

Las relaciones oxígeno/coque de 0.94 y 0.77 son las relaciones donde el CRC y el CRM, respectivamente, alcanzan su máxima eficiencia térmica con un valor de $83.22 \%$ y $86.18 \%$, la primera para una alimentación de coque CRC de $6418.304 \mathrm{~kg} / \mathrm{h}$ y la segunda para una alimentación de coque de CRM de $7818.304 \mathrm{~kg} / \mathrm{h}$.

El coque de petróleo a pesar de ser considerado un desecho de refinería su precio se incrementará de acuerdo a la demanda de éste para generar un gas sintético capaz de usarse como combustible.

El conocimiento del rendimiento del coque de petróleo usando la tecnología de la gasificación resulta de importancia para estimar o evaluar la factibilidad y conveniencia de integrar un sistema de gasificación de los coques mexicanos a una planta de Ciclo Combinado para la generación limpia de electricidad, considerando a la tecnología de los ciclos combinados con gasificación integrada como una alternativa viable de implementarse en México.

Finalmente, las simulaciones numéricas suministran información detallada y completa relacionada con balances de masa y energía, usualmente con mayor resolución y flexibilidad que las logradas con los trabajos experimentales.

\section{Agradecimientos}

A la División de Estudios de Posgrado e Investigación del Instituto Tecnológico de Cd. Madero por el apoyo recibido para la realización de esta investigación.

\section{Referencias}

Ahman S, Pisano-Altom J, Charly Tsiou G.C. Refinería de Taiwan utilizará coque de petróleo en dos CFB's de avanzada tecnología. Power. Mayo/junio 2002.

Anthony E.J., Anderson K., Carson R., Laub I.I. Petroleum Coke and Coal Start up Testing in Bubbling Fluidized Bed Combustor. Transaction of The ASME, Journal of Energy Resources Technology, (119): 96-109. junio 1997.

ASPEN PLUS SHELL. Aspen Technology Inc. 2003.

Bejan A. Advanced Engineering Thermodinamics. Wiley-Interscience publication. John Wiley and Son. 1980.

Bolado-Estadía R. Nuevas tecnologías para la utilización de combustibles fósiles en la generación eléctrica. Publicación Interna. Institu- to de Investigaciones Eléctricas (IIE). Cuernavaca, México. 1995. Pp. 1-41.

Bryan S. Bock H.Y., Rosenquist W.A. Petroleum Coke as a Viable Alternative Fuel. POWER-GEN International. Orlando, Florida. EUA. 1996, pp. 259-267.

Cengel-Yunus A. y Boles-Michael A. Thermodinamics: An Engineering Approach. 3a. Ed. McGraw-Hill. ISBN 0-07-11524-4. 1998.

Cook C.S., Corman J.C., Todd D.M. System Evaluation and LBTU Studies for IGCC Power Generation. Journal of Engineering for Gas Turbines and Power, 117:673-677. October 1995.

EPRI, Final Report, 2006 [en línea]. Petcoke and Low-Rank Coal/ Lignite Supply Outlook for IGCC Evaluations. Program 066.0 Future Coal Generation Options, 1013038 Final Report 200602. Disponible en: http://www.epri.com/OrderableitemDesc. asp?product_id $=000000000001013038 \&$ searchdate $=02 / 22 / 2006$

Fabela R. Alternativas para la utilización del coque. Instituto de Investigaciones Eléctricas (IIE), workshop de carbón. Subdirección de Planeación de Pemex-Refinación. Febrero 1998.

Fernández-Montiel M. y Alaniz-Chávez F.J. Gasificación de combustibles sólidos y de combustóleo para la generación eléctrica. Reporte del estado actual de las tecnologías de gasificación aplicadas a la generación de energía eléctrica. Reporte No. 44207, IIE, División de sistemas mecánicos. Departamento de Ingeniería Mecánica. 1995.

Fernández-Montiel M., González-Santaló J.M., Gutiérrez-Ramírez R., Milán-Foressi J. y Romo-Millares C. Tendencias tecnológicas en los procesos de combustión en la generación de electricidad. Boletín IIE. Mayo/junio 1998.

Fernández-Montiel M, Manzanares E., Altamirano J. y Mani A. Estudio de factibilidad de combustibles a gasificar en plantas IGCC. Instituto de Investigaciones Eléctricas. Informe IIE/44/ 13043/1002/P-México, 2007.

Fernández-Montiel M. Tendencias tecnológicas: nuevos combustibles (coque de petróleo, carbón y emulsiones). Boletín Instituto de Investigaciones Electricas (IIE), octubre 2008.

Generux R.P. y Doucette B. Pet-Coke-Firing Experience Envolves Over three Decades. Power, 7(140):61-62. July/august, 1996.

González-Fernández R. y Villalobos H. Procesamiento de crudos pesados en México. [en línea] Instituto Mexicano del Petróleo (IMP), 2001. Disponible en: www.imiq.org/leon99/memorias/ trabajos/Jt8/jt8-4.htm.

González-Rocha J.C. Estudio del desempeño de una planta de potencia de ciclo combinado con gasificación integrada. (Tesis doctoral). Centro Nacional de Investigación y Desarrollo Tecnológico. Departamento de Ingeniería Mecánica, 2007.

González-Rocha J.C., Longoria-Ramírez R., Urquiza-Beltrán G. Resultados de la simulación de gasificación del coque de petróleo de las refinerías de Ciudad Madero y Cadereyta. Ingeniería Investigación y Tecnología. IX(2):99-111. 2008.

Himan C. y Vander-Burgt M. Gasification. Editorial Elsevier Science USA. British library Cataloging-in-Publication Data. ISBN0-7506-7707-4. 2003. 
Holt N. Gasificación de combustibles fósiles. Boletín Instituto de Investigaciones Eléctricas (IIE), tendencias tecnológicas. Noviembre-diciembre, 1996. Pp. 256-259.

Hui-Zou J., Jie-Zhou Z., Chen-Wang F., Zhang W., Hua-Dai Z., Feng-Liu H., Hong-Yu Z. Modeling Reactor Kinetics of Petroleum Coke Gasification with $\mathrm{CO}_{2}$. Chemical Engineering and Processing, 2006.

Johnk, C.B., Abdulally I.F. Experience Firing Petroleum Coke-An Udate, Foster Wheeler Energy International, Inc., 1996.

Mahagaokar U. y Hauser N. Gasification of Petroleum Coke in the Shell Coal Gasification Process. Fifth International Power Generation Exibition \& Conference. POWER-GEN (5 de noviembre 1992, Orange Country C. Orlando Florida). 1992. Pp. 17-19.

Millán F. y Julio J. La combustión de coque de petróleo en un combustor experimental de lecho fluidizado. Congreso Internacional de Calderas y Recipientes a Presión (XV, 1992,
Guadalajara, Jalisco) Instituto de Investigaciones Eléctricas. Depto. de Ingeniería Mecánica. México, Noviembre 1992.

Nagpal S., Sarkar, T.K., Sen P.K. Simulation of Petcoke Gasification in Slagging Moving Bed Reactors. Fuel processing Technology ELSEVIER, pp. 617-640. 2004.

Northridge-Phillips J. A Study of the Off-Design Performance of Integrated Coal Gasification Combined-Cycle Power Plants. Thesis (Ph. D.), University de Stanford, 1986.

Pemex Refinación. Incremento en la capacidad de refinación [en línea] Abril 2009. Disponible en: www.pemex.com/files/content/capacidad refinación1.pdf

Secretaría de energía [en línea]. Publicaciones 2007. Prospectiva petrolíferas 2007-2016. Disponible en: www.energía.gob.mx

Yañez F. Petcoke: Combustible o desecho. Potencia 6.3, mayo-junio 2000, pp. 38-40.

\section{Semblanza de los autores}

José Clemente González-Rocha. Es doctor en ciencias (ingeniería mecánica) por el Centro Nacional de Investigación y Desarrollo Tecnológico (CENIDET). Desde 1991 ha trabajado como profesor titular para la DGEST, desarrollando proyectos de investigación en el campo de termo-fluidos y plantas de potencia. Actualmente, pertenece a la División de Posgrado e Investigación del Instituto Tecnológico de Cd. Madero (ITCM).

Gustavo Urquiza-Beltrán. Es doctor en ingeniería mecánica por la Escuela Politécnica de Montreal, Canadá en 1995. Desde 1985, ha trabajado en proyectos de investigación relacionados con termo-hidráulica y turbomaquinaria en el Instituto de Investigaciones Eléctricas, en el Centro de Investigación y Desarrollo Tecnológico (CENIDET) y en el Centro de Investigación en Ingeniería y Ciencias Aplicadas (CIICAp) de la Universidad Autónoma del Estado de Morelos. Actualmente es director del CIICAp y pertenece al Sistema Nacional de Investigadores (SNI).

Rigoberto Longoria-Ramírez. Es doctor por la Universidad de Aberdeen, Escocia, Gran Bretaña. Ha trabajado en proyectos de investigación para el Instituto de Investigaciones Eléctricas (IIE), el centro de Ciencias de la Atmósfera de la UNAM, el Instituto Nacional de Ecología en campos relacionados con procesos químicos, contaminación ambiental y aprovechamiento de fuentes alternas de energía. Pertenece al Sistema Nacional de Investigadores (SNI) desde el año de 1989 y actualmente es profesor en el centro de investigación y desarrollo tecnológico (CENIDET). 